\title{
Recombinant prolylcarboxypeptidase activates plasma prekallikrein
}

\section{Hall 5 09:30 15th J uly, 2003}

Session Type: Oral communications

Subject area: Contact phase

Session title: Prekallikrein and kininogens

Abstract: $0 \mathrm{C} 121$

Authors: Z. Shariat-Madar*, F. Mahdi, ${ }^{*} \&$ A. H. Schmaier*

*University of Michigan, USA

Recently, we recognized that the serine protease prolylcarboxypeptidase (PRCP), an angiotensin II degrading enzyme, isolated from HUVEC is a plasma prekallikrein (PK) activator. These data indicate that there is a physiologic basis for activation of the proteins of the plasma kallikrein/kinin system (KKS) when assembled on HUVEC membranes. Present investigations determined if recombinant PRCP ( $\mathrm{rPRCP}$ ) activates PK. PRCP cDNA was cloned in pMT/BIP/V5-HIS-C followed by transient transfection into S2 Schneider insect cells and purified from serum-free media. Mature $\mathrm{rPRCP}$, but not that which has the propeptide, activated PK when bound to high molecular weight kininogen (HK) linked to microtiter plates. Recombinant PRCP's ability to activate PK was inhibited by antipain (IC50 $=3 \mu \mathrm{M})$, leupeptin $(\mathrm{IC50}=3 \mu \mathrm{M}), \mathrm{KPI}$ domain of the amyloid precursor protein $(\mathrm{IC} 50=60 \mu \mathrm{M})$, and corn trypsin inhibitor $(\mathrm{IC50}=10 \mathrm{nM})$, but not by 1-3 mM EDTA, o-phenanthroline, iodoacetamide, or iodoacetic acid. Angiotensin II or bradykinin (BK), but not angiotensin II (1-7) or BK (1-5), inhibited PK activation with an IC50 $=0.2$ or $0.1 \mathrm{mM}$, respectively. These findings are similar to PRCP isolated from HUVEC. AntiPRCP inhibited rPRCP's ability to activate PK. Unlike factor XIIa, rPRCP's ability to activate PK is blocked by angiotensin II but not neutralizing antibody to factor XIIa. The $\mathrm{Km}$ of $\mathrm{rPRCP}$ activation of PK was $17 \mathrm{nM}$. PRCP antisense oligonucleotide inhibited PRCP activity on HUVEC, indicating that PRCP is the major PK activating enzyme. On flow cytometry, PRCP is specifically found on the membrane of nonpermeabilized HUVEC in suspension. On laser scanning confocal microscopy, both PRCP and LAMP1 are on the membrane of nonpermeabilized cells. When permeabilized, PRCP and LAMP1 antigen do not co-localize in lysosomes. On the membrane of fixed but not permeabilized HUVEC, PRCP co-localizes with all of the $\mathrm{HK}$ binding proteins in the following order: gClqR, UPAR, and cytokeratin 1. PRCP appears to be the major PK activator on HUVEC. These data support the hypothesis that the KKS counterbalances the renin-angiotensin system. The interaction of these systems may serve as a direct link between blood pressure control and thrombosis in vivo. 
Supplement to the J ournal of Thrombosis and Haemostasis J uly 2003 (I SSN 1740 - 3340) 